\title{
Many-Body Effects Observed in the Positron Annihilation Experiment
}

\author{
G. KONTRYM-SZnAJd ${ }^{a}$ AND H. SORMANN ${ }^{b}$ \\ ${ }^{a}$ Institute of Low Temperature and Structure Research \\ Polish Academy of Sciences, P.O. Box 937, 50-950 Wrocław 2, Poland \\ ${ }^{b}$ Institut für Theoretische Physik - Computational Physics \\ Technische Universität Graz, Petersgasse 16, 8010 Graz, Austria \\ This paper is devoted to study many-body effects in the positron annihi- \\ lation experiment, both electron-positron ( $\mathrm{e}-\mathrm{p})$ and electron-electron (e-e) \\ correlations. Various theories of the e-p interaction in real solids were used \\ to verify them by comparing theoretical and experimental $\mathrm{e}-\mathrm{p}$ momentum \\ densities in $\mathrm{Cu}$ and $\mathrm{Y}$. We show that the lattice potential has an essential \\ influence on the e-p correlation effects, i.e. their proper description must \\ be done via periodic lattice potential as e.g. in the Bloch modified ladder \\ theory. Moreover, it is not true that the dynamic parts of the direct $\mathrm{e}-\mathrm{p}$ \\ and e-e interactions cancel each other because e-e correlations are observed \\ not only in the Compton scattering but also in the positron annihilation \\ experiments.
}

PACS numbers: 78.70.Bj, 78.70.Ck, 74.25.Jb

\section{Introduction}

The first question considered in the paper is connected with the electron-positron interaction in real metals. It is shown that the Bloch modified ladder (BML) theory [1], in contrast to all other approaches, is able to describe (at least qualitatively) experimental e-p momentum densities for both simple and transition metals. Such a finding is important because all these theories, except BML, ignore the influence of the lattice potential on the e-p interaction, concerning both intraband and interband transitions.

The next subject of the paper are the electron-electron correlations which should, in principle, be also observed in the positron annihilation experiments. However, Carbotte and Kahana [2] showed that (at least for positrons in jellium) the dynamical parts of the e-p and e-e correlation cancel each other. Consequently, the remaining many-body effects come only from the static part of these interactions. Because the static e-e correlations are (at least approximately) included 
into the band structure calculations, almost all positron annihilation theories consider only the static part of the e-p correlations, based on the result of Carbotte and Kahana [2]: an annihilating e-p pair is, seen from outside, a neutral quantity with a strongly reduced coupling to its environment ([3] and refs. therein). The resulting enhancement factor is strongly momentum-dependent and leads to a monotonously increasing e-p momentum density below the Fermi momentum $p_{\mathrm{F}}$, an effect which we call Kahana-like enhancement. Beyond that, there exists only one electron gas theory by Arponen and Pajanne [4] where the e-e interaction, on the level of the well-known random phase approximation (RPA), is described by non-interacting Sawada bosons, and each boson-boson interaction goes beyond the RPA. Contrary to the result of Ref. [2], Arponen and Pajanne observed a significant tail of the $\mathrm{e}-\mathrm{p}$ momentum density beyond $p_{\mathrm{F}}$ due to dynamical $\mathrm{e}-\mathrm{e}$ and e-p correlations. However, according to the results in Ref. [4], the values of the enhancement factor (EF) on the Fermi surface (FS) increase with the increasing density of the electron gas. Since such a behaviour of the EF strongly contradicts the experiments, all theories of the e- $\mathrm{p}$ annihilation [3] are based on the results of Kahana and Carbotte [2].

According to our knowledge, the existence of many-body tails in positron annihilation data has been observed for the first time by Manuel et al. [5] for Sn- $\beta$ and even for such jellium-like metals as Li and Al. Next, Ohata et al. [6] compared a high-resolution Compton profile (CP) with one-dimensional (1D) angular correlation of annihilation radiation (ACAR) spectrum along [111] direction in Al. They observe a Kahana-like enhancement near the FS and a weaker tail of densities for $p>p_{\mathrm{F}}$ in the case of the 1D ACAR data compared to the CPs, as a consequence of the partial cancellation of $\mathrm{e}-\mathrm{e}$ and $\mathrm{e}-\mathrm{p}$ correlations. Here we would like to point out that the BML theory, applied to $\mathrm{Al}[1 \mathrm{a}]$, gives the following results. Whereas the enhancement factor for momenta $p<p_{\mathrm{F}}$ is similar to the Kahana-like enhancement, for $p>p_{\mathrm{F}}$ the contribution of Umklapp components is significantly diminished by the e- $\mathrm{p}$ interaction. Moreover, it reduces (in comparison with independent particle model (IPM)) the core contribution as well as the enhancement factor for core electrons decreases for higher momenta. Therefore, a weaker tail for $p>p_{\mathrm{F}}$ observed in $\mathrm{Al}$ [4] could be connected with these e-p correlation effects (not with weaker e-e correlations as interpreted in [6]). A simultaneous analysis of both reconstructed densities and $1 \mathrm{D}$ profiles for Compton scattering and 2D ACAR experiment in $\mathrm{Y}$, allowed us to state that in this material e-e correlations in the ACAR data are exactly the same as in the Compton scattering experiment [7], an effect which has been recently observed also in $\mathrm{LaB}_{6}$ [8] and in $\mathrm{Mg}$ [9].

\section{Applied theories}

In the ACAR or the Compton scattering experiments one measures integrals of the electron-positron or electron momentum densities in the extended $\boldsymbol{p}$ space, respectively 


$$
\rho(\boldsymbol{p})=\sum_{j} n_{\boldsymbol{k} j}\left|\int_{-\infty}^{\infty} \mathrm{e}^{\mathrm{i} \boldsymbol{p} \cdot \boldsymbol{r}} \psi_{\boldsymbol{k} j}^{\mathrm{e}-\mathrm{p}}(\boldsymbol{r}, \boldsymbol{r}) \mathrm{d} \boldsymbol{r}\right|^{2},
$$

where $n_{\boldsymbol{k} j}$ is the occupation number (0 or 1 ) of the electron Bloch state $\boldsymbol{k} j$ and $\psi_{\boldsymbol{k} j}^{\mathrm{e}-\mathrm{p}}(\boldsymbol{r}, \boldsymbol{r})$ is the pair wave function of an electron and a thermalized positron.

We used five models for the wave function of an $\mathrm{e}-\mathrm{p}$ pair moving within a lattice-periodical crystal potential, where for the numerical evaluation of the electron and positron wave functions, the augmented plane-wave (APW) method has been applied (details in [10]):

1. $\psi_{\boldsymbol{k} j}^{\mathrm{e}-\mathrm{p}}(\boldsymbol{r}, \boldsymbol{r})=\psi_{\boldsymbol{k} j}(\boldsymbol{r})$ - the electron momentum density (EMD).

2. $\psi_{\boldsymbol{k} j}^{\mathrm{e}-\mathrm{p}}(\boldsymbol{r}, \boldsymbol{r})=\psi_{\boldsymbol{k} j}(\boldsymbol{r}) \psi_{+}(\boldsymbol{r})$ - the IPM.

3. $\psi_{\boldsymbol{k} j}^{\mathrm{e}-\mathrm{p}}(\boldsymbol{r}, \boldsymbol{r})=\sqrt{g(\boldsymbol{r}, \boldsymbol{k} j)} \psi_{\boldsymbol{k} j}(\boldsymbol{r}) \psi_{+}(\boldsymbol{r})$ - the most popular approach, where a local e-p correlation function $g$ is inserted into IPM formula. In the present paper, for $g$ the following cases were considered:

3.1. the local-density approximation (LDA), as proposed by Daniuk et al. [11] where $g(\boldsymbol{r} ; j \boldsymbol{k})=\varepsilon_{\mathrm{hom}}\left(r_{\mathrm{s}}(\boldsymbol{r}) ; \chi_{j \boldsymbol{k}}\right)$ with $r_{\mathrm{s}}(\boldsymbol{r})$ as the local density parameter. The enhancement factors $\varepsilon_{\text {hom }}$ are results of an $\mathrm{e}-\mathrm{p}$ enhancement theory for the electron gas [12]. Here the correlation function is state-dependent (we call it state-dependent LDA) where $\chi_{n k}=\left[\left(E_{j \boldsymbol{k}}-E_{0}\right) /\left(E_{\mathrm{F}}-E_{0}\right)\right]^{1 / 2}$ with $E_{0}, E_{\mathrm{F}}$, and $E_{j \boldsymbol{k}}$ as: the bottom energy of the electron conduction bands, the Fermi energy, and electron energy in the Bloch state $|j \boldsymbol{k}\rangle$, respectively.

3.2. An LDA-type theory which neglects the explicit momentum-dependence of correlation function $g$ (we call it state-independent LDA) [13]. As before, $\varepsilon_{\text {hom }}$ is taken for homogeneous electron gases (we applied the formula of Boroński and Nieminen [14]).

4. The so-called BML theory [1a], based on an earlier paper of Carbotte [1c] and Fujiwara $[1 \mathrm{~b}]$ where the e-p interaction is included via a lattice-periodic crystal potential.

Other theories, used for describing the e-p interaction in real metals (being similar to theories described in the point 3) are the following: the weighted density approximation (WDA) [15], the generalized gradient approximation (GGA) [16] and the theory proposed by Alatalo et al. and Barbiellini et al. [17] where the correlation function $g$ is substituted by the state dependent correlation factor $\gamma_{\boldsymbol{k} j}$. However, this state dependence $\boldsymbol{k}$ is not connected with either energy or momentum dependence as given in the function $g$. It follows from a state dependence of the ratio $\gamma_{\boldsymbol{k} j}=\lambda_{\boldsymbol{k} j} / \lambda_{\boldsymbol{k} j}^{\mathrm{IPM}}$, where $\lambda$ denotes the local annihilation rates which could be calculated within state independent either LDA or GGA.

\section{Results}

In this section we present theoretical e-p momentum densities $\rho(p)$ for yttrium and copper, compared with densities reconstructed from both $2 \mathrm{D}$ ACAR 
spectra (for $\mathrm{Y}$ and $\mathrm{Cu}$ ) and $1 \mathrm{D}$ high-resolution Compton profiles (for $\mathrm{Y}$ ). 2D ACAR spectra represent line projections of e-p momentum densities while 1D CPs plane projections of electron densities, both densities are studied in the extended $\boldsymbol{p}$ space.

Five profiles for $\mathrm{Y}$ were measured with overall resolution about 0.15 atomic units of momentum (a.u.), using the 2D-ACAR spectrometer at the University of Texas at Arlington [18]. Next, e-p densities $\rho(\boldsymbol{p})$ were reconstructed by applying the Cormack method [19]. e-p densities for $\mathrm{Cu}$, reconstructed from six experimental 2D ACAR spectra, were taken from Ref. [20]. Experimental electron momentum densities for $\mathrm{Y}$ were reconstructed from 12 high-resolution CPs, measured with overall resolution about 0.16 a.u. at the European Synchrotron Radiation Facility (ESRF), France - more details in [7].

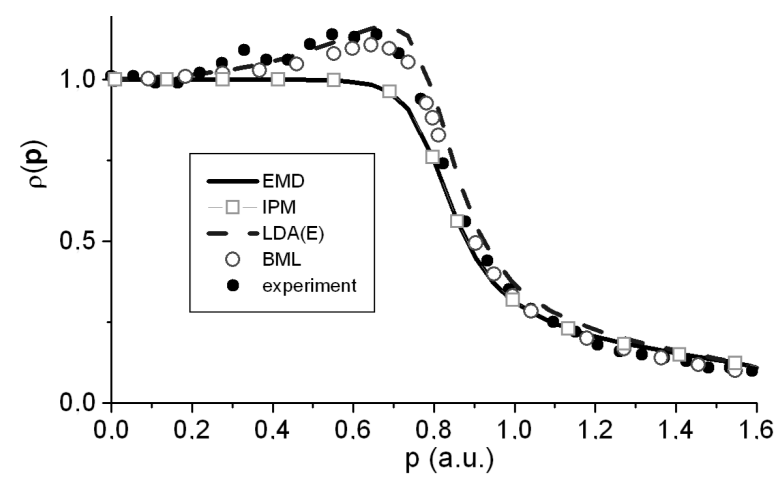

Fig. 1. Theoretical e-p momentum densities for copper along [111], compared with densities reconstructed from 2D ACAR data [20].

In Fig. 1 we show results for $\mathrm{Cu}$ where the reconstructed densities show a typical Kahana-like enhancement. This behaviour can be satisfactorily described by the state-dependent LDA and the BML theory, in contrast to the other theories mentioned in the previous section.

In Fig. 2 we present reconstructed e-p densities in $\mathrm{Y}$ along the direction $\Gamma M$ on the basal $\Gamma M K$ plane, compared with theoretical results. Presented theoretical results are not convoluted (not smeared by the experimental resolution) while reconstructed densities are after applying Max Entropy deconvolution procedure $[18,21]$. It is evident that the EMD is essentially different from the electron density "observed" by the positron, particularly for the high-momentum region. This behaviour reflects the well-known fact that high-momentum contributions to the momentum density are significantly reduced by the appearance of a positron.

For yttrium, the application of the IPM leads to a momentum profile which is very similar to the BML result (drawn by open circles), and both curves fit the reconstructed data rather well. The inclusion of $\mathrm{e}-\mathrm{p}$ correlation effects shows the following behaviour: the use of both a local and state-dependent correlation function (according to the proposal by Daniuk et al. [11], see the dashed curve 


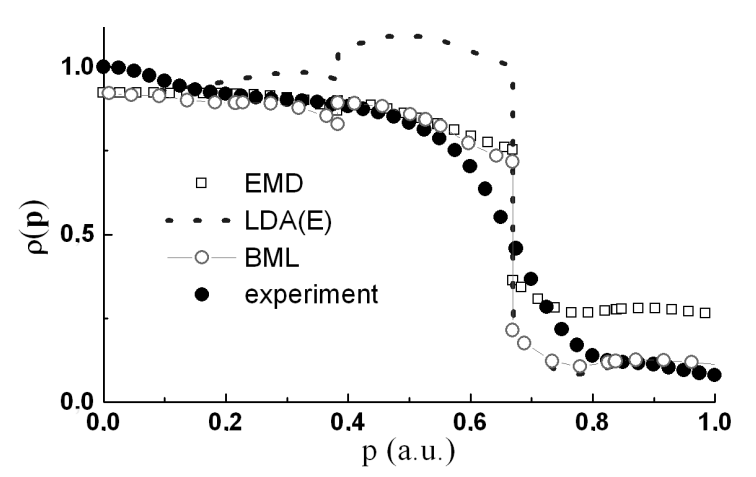

Fig. 2. Theoretical e-p momentum densities in $\mathrm{Y}$ along $\Gamma M$, compared with densities reconstructed from $2 \mathrm{D}$ ACAR experimental spectra.

in Fig. 2) leads to a strongly increased momentum density. It is a typical $s p$-like enhancement, well-known for metals with marked nearly-free valence electrons like in alkalis, aluminium, or even copper [20]. However, for yttrium such a behaviour is in clear contrast to the (reconstructed) experimental densities. This is somewhat surprising, taking into account that the $5 s+4 d$ electrons in yttrium are far from the ions and should therefore be considered by the positrons as nearly-free particles (especially the electrons from the 1st and 2nd valence band where $E=E(\boldsymbol{k})$ is close to the parabolic function). The best agreement between theory and experiment can be obtained by including the e-p correlation effects according to a state-independent LDA with a local enhancement function, or by the application of the BML theory, particularly in those regions where contributions from the partially occupied 3rd and 4th band dominate. Still remaining differences between theory and experiment lead to the conclusion that contributions of Umklapp components of the electron wave function are stronger decreased by the positron than it follows from applied theories.

There is also no experimental indication for a Kahana-like enhancement $[22,23]$ in a typical transition metal like Cr. Similarly as in Y, the momentum density decreases monotonously with increasing moment, and the density values yielded by a state-dependent LDA or by WDA [23] theory are too high, whereas other theories like state-dependent LDA or the BML approach lead to results which fit experiment rather well.

In Ref. [7], we recently published further results of $\mathrm{Y}$, based on a simultaneous analysis of yttrium 2D-ACAR data and of high-resolution CPs which allowed us the observation of strong e-e correlation effects in the positron annihilation data. Theoretically, e-e correlations were taken into account following the work of Cardwell and Cooper [24] based on the proposal of Lam and Platzman [25], where the Lam-Platzman (LP) corrections have been calculated from the self-consistent APW electron charge density. Studying both a directional anisotropy of measured spectra and reconstructed densities, we obtained that in the case of 


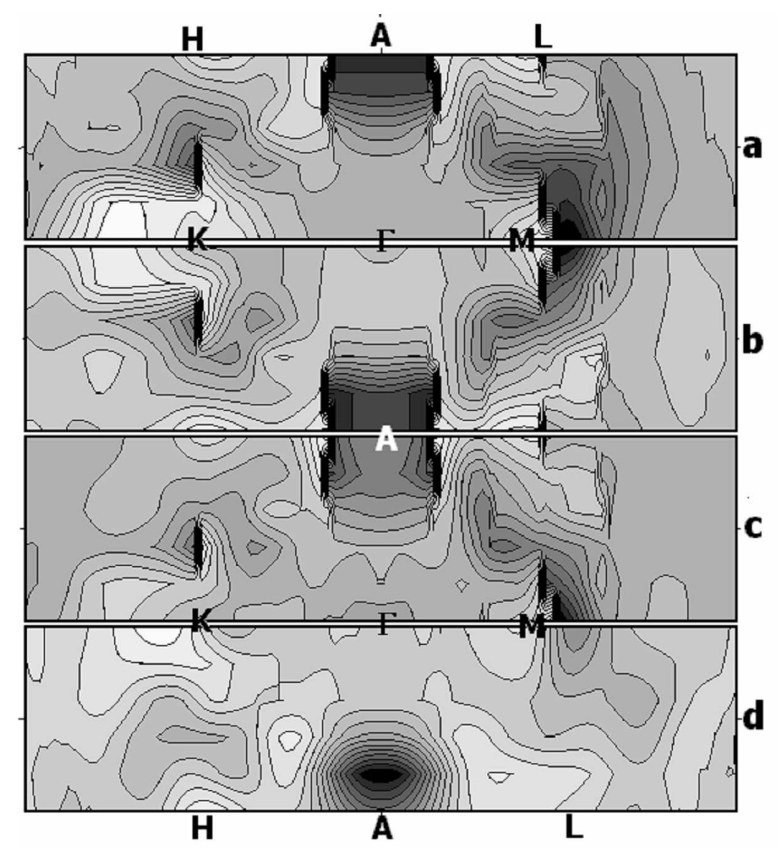

Fig. 3. Anisotropic part of electron momentum densities in $\mathrm{Y}$ along $\Gamma M, \Gamma K$ and parallel directions (for momenta up to 1.37 a.u.) for: (a) pure theoretical EMD densities; differences between theoretical and experimental densities: (b) $\rho^{\mathrm{EMD}}(\boldsymbol{p})-\rho^{\mathrm{CP}}(\boldsymbol{p})$; (c) $\rho^{\mathrm{IPM}}(\boldsymbol{p})-\rho^{\mathrm{ACAR}}(\boldsymbol{p}) ;(\mathrm{d})$ densities reconstructed from convoluted theoretical and experimental CPs.

the Compton profiles there are strong e-e correlations which cannot be described by such isotropic e e Lam-Platzman corrections - this effect is seen in Fig. 3b. However, it was even more surprising that we got exactly the same differences between theoretical and reconstructed experimental densities $\rho^{\mathrm{EMD}}(\boldsymbol{p})-\rho^{\mathrm{CP}}(\boldsymbol{p})$ and $\rho^{\mathrm{IPM}}(\boldsymbol{p})-\rho^{\mathrm{ACAR}}(\boldsymbol{p})$ (in $\mathrm{Y} \rho^{\mathrm{IPM}}(\boldsymbol{p})$ is almost the same as $\rho^{\mathrm{BML}}(\boldsymbol{p})$, i.e. e-p correlations do not change momentum dependence of densities). Therefore, it is clear that also the e-p momentum density is strongly influenced by the e-e correlations, presented clearly in Fig. 3d. This finding was surprising because almost all theories devoted to this question [3] are based on the result of Carbotte and Kahana [2] where e-p pair is, seen from outside, a neutral quantity with a strongly reduced coupling to its environment. Consequently, typical correlation effects as smearing at the Fermi momentum and high-momentum tails of the momentum distribution should be significantly smaller than in pure electron systems. However, a detailed analysis of $2 \mathrm{D}$ ACAR spectra show that it is not true.

\section{Conclusions}

By applying various models of the $\mathrm{e}-\mathrm{p}$ wave function, we found out that the $\mathrm{e}-\mathrm{p}$ momentum density in simple metals as $\mathrm{Al}$, with its typical Kahana-like 
enhancement, can be satisfactorily described by the use of state-dependent enhancement theories like state-dependent LDA or WDA. On the other hand, such theoretical approaches completely fail in the case of transition metals like $\mathrm{Cr}$ or $\mathrm{Y}$ where methods like the state-independent LDA succeed. Only the BML theory works reasonably well for both simple and transition metals. Our explanation for this behaviour is as follows.

In the Kahana formalism [2] the e-p wave function is given by

$$
\psi_{\mathrm{p}}^{\mathrm{e}-\mathrm{p}}\left(x_{\mathrm{e}}, x_{\mathrm{p}}\right)=\exp \left(\mathrm{i} p \cdot x_{\mathrm{e}}\right)+\sum_{\tilde{p}>p_{\mathrm{F}}} \zeta(p, \tilde{p}) \exp \left(\mathrm{i} \tilde{p} \cdot x_{\mathrm{e}}\right) \exp \left[\mathrm{i}(p-\tilde{p}) \cdot x_{\mathrm{p}}\right],
$$

where function $\zeta(p, \tilde{p})$ describes a perturbation (due to the e-p interaction) of the free-electron state $p$. Due to the Pauli principle, in the case of the electron gas where all states inside the FS are fully occupied, perturbed states can be described only by $\tilde{p}>p_{\mathrm{F}}$. Resulting enhancement factor is growing with $p$, having maximal values at the FS. Therefore, such Kahana-like enhancement is for the case when all states inside the FS are fully occupied (the probability of scattering is the highest for electrons at the FS). However, in real solids, due to the lattice potential, for each occupied band, there is always a leading term of density (where the occupation number is lower than 1) and the Umklapp components. Therefore, one could expect the following: the higher the lattice effects are the weaker is the Kahana-like $p$ dependence of the enhancement. This fact is an inherent feature of the BML theory where the e-p interaction matrix is based on electron Bloch eigenstates. Therefore, in this theory, the influence of the crystal lattice on e-e and $\mathrm{e}-\mathrm{p}$ scattering processes is more realistically described than in other theoretical approaches. Moreover, it is not true that the dynamic parts of the direct e-p and e-e interactions cancel each other and in the positron annihilation experiment one should observe only the static part of the e-p interaction. e-e correlations are not cancelled, they are strong (as in the Compton scattering experiment [26]) and they cannot be described by the isotropic LP correction [25].

\section{Acknowledgments}

We are very grateful to the State Committee for Scientific Research (Poland) (grant 2 P03B 012 25) for the financial support.

\section{References}

[1] H. Sormann, Phys. Rev. B 54, 4558 (1996); K. Fujiwara, Phys. Soc. Jap. 33, 1047 (1972); J.P. Carbotte, Phys. Rev. 144, 309 (1966).

[2] J.P. Carbotte, S. Kahana, Phys. Rev. 139, A213 (1965).

[3] M. Šob, H. Sormann, J. Kuriplach, Adv. Quant. Chem. 42, 77 (2003); A. Rubaszek, Z. Szotek, W.M. Temmerman, Phys. Rev. B 65, 125104 (2002).

[4] J. Arponen, E. Pajanne, Ann. Phys. (New York) 121, 343 (1979); J. Phys. F 9, 2359 (1979). 
[5] A.A. Manuel, D. Vasumathi, B. Barbiellini, A. Shukla, P. Suortti, T. Chiba, Mater. Sci. Forum 255-257, 760 (1997).

[6] T. Ohata, M. Itou, I. Matsumoto, Y. Sakurai, H. Kawata, N. Shiotani, S. Kaprzyk, P.E. Mijnarends, A. Bansil, Phys. Rev. B 62, 16528 (2000).

[7] G. Kontrym-Sznajd, M. Samsel-Czekała, A. Pietraszko, H. Sormann, S. Manninen, S. Huotari, K. Hämäläinen, J. Laukkanen, R.N. West, W. Schülke, Phys. Rev. B 66, 155110 (2002).

[8] G. Kontrym-Sznajd, M. Samsel-Czekala, M. Biasini, Y. Kubo, Phys. Rev. B 70, 125103 (2004).

[9] G. Kontrym-Sznajd, M. Samsel-Czekała, Acta Phys. Pol. A 107, 586 (2005).

[10] H. Sormann, Phys. Rev. B 43, 884 (1991).

[11] S. Daniuk, G. Kontrym-Sznajd, A. Rubaszek, H. Stachowiak, J. Mayers, P.A. Walters, R.N. West, J. Phys. F 17, 1365 (1987).

[12] A. Rubaszek, H. Stachowiak, Phys. Rev. B 38, 3846 (1988).

[13] S. Daniuk, M. Šob, A. Rubaszek, Phys. Rev. B 43, 2580 (1991).

[14] E. Boroński, R.M. Nieminen, Phys. Rev. B 34, 3820 (1986).

[15] A. Rubaszek, Z. Szotek, W.M. Temmerman, Phys. Rev. B 58, 11285 (1998).

[16] B. Barbiellini, M.J. Puska, T. Torsti, R.M. Nieminen, Phys. Rev. B 51, 7341 (1995).

[17] M. Alatalo, B. Barbiellini, M. Hakala, H. Kauppinen, T. Korhonen, M.J. Puska, K. Saarinen, P. Hautojärvi, R.M. Nieminen, Phys. Rev. B 54, 2397 (1996); B. Barbiellini, M. Hakala, M.J. Puska, R.M. Nieminen, A.A. Manuel, Phys. Rev. B 56, 7136 (1997).

[18] S.B. Dugdale, H.M. Fretwell, M.A. Alam, G. Kontrym-Sznajd, R.N. West, S. Badrzadeh, Phys. Rev. Lett. 79, 941 (1997).

[19] A.M. Cormack, J. Appl. Phys. 35, 2908 (1964); G. Kontrym-Sznajd, Phys. Status Solidi A 117, 227 (1990).

[20] F. Sinclair, W.S. Farmer, S. Berko, Positron Annihilation, Eds. P.G. Coleman, S.C. Sharma, L.M. Diana, North-Holland, Amsterdam 1982, p. 322.

[21] S.B. Dugdale, M.A. Alam, H.M. Fretwell, M. Biasini, D. Wilson, J. Phys., Condens. Matter 6, L435 (1994); H.M. Fretwell, S.B. Dugdale, M.A. Alam, M. Biasini, L. Hoffmann, A.A. Manuel, Europhys. Lett. 32, 771 (1995).

[22] H. Sormann, G. Kontrym-Sznajd, R.N. West, Mater. Sci. Forum 363-365, 609 (2001).

[23] A. Rubaszek, Z. Szotek, W.M. Temmerman, Phys. Rev. B 65, 125104 (2002).

[24] D.A. Cardwell, M.J. Cooper, J. Phys.: Condens. Matter 1, 9357 (1989).

[25] L. Lam, P.M. Platzman, Phys. Rev. B 9, 5122 (1974).

[26] G. Stutz, F. Wohlert, A. Kaprolat, W. Schülke, Y. Sakurai, Y. Tanaka, M. Ito, H. Kawata, N. Shiotani, S. Kaprzyk, A. Bansil, Phys. Rev. B 60, 7099 (1999); S. Huotari, K. Hämäläinen, S. Manninen, S. Kaprzyk, A. Bansil, W. Caliebe, T. Buslaps, V. Honkimäki, P. Suortti, Phys. Rev. B 62, 7956 (2000); H. Reniewicz, A. Andrejczuk, M. Barancewicz, E. Żukowski, L. Dobrzyński, S. Kaprzyk, Phys. Status Solidi B 241, 1849 (2004). 